\title{
Potential of combating transmission of COVID-19 using novel self-cleaning superhydrophobic surfaces: part I- protection strategies against fomites
}

\author{
S. A. Meguid $(\mathbb{D} \cdot$ Assem Elzaabalawy $\mathbb{D}$
}

Received: 13 July 2020/Accepted: 27 July 2020/Published online: 5 August 2020

(C) Springer Nature B.V. 2020

\begin{abstract}
According to the World Health Organisation, one of the main concerns of COVID-19 virus is its tenacity to spread from droplets that either land directly on a surface or are transmitted to a surface by an infected person. In this study, we report the potential of using superhydrophobic surfaces to combat the transmission and spread of fomites infected by COVID-19 virus strand. Fomites include clothes, utensils, furniture, regularly touched objects and personal protective equipment used by Health Care Workers to act as barriers against fluid transmission and/or fluid penetration. In this effort, we propose three strategies to combat the transmission and the spread of the virus: encapsulation, contamination suppression, and elimination. We believe that this can be achieved by the use of our recently developed superhydrophobic coating and regenerative monolith to encapsulate and suppress the virus. The newly developed superhydrophobic coating and monolith are scalable, economical, and facile with the monolith capable of regeneration. The elimination of the virus will be through the use of antiviral and antibacterial copper nanoparticles or dedicated copper surfaces.
\end{abstract}

S. A. Meguid $(\varangle) \cdot$ A. Elzaabalawy

Mechanics and Aerospace Design Lab, University of

Toronto, 5 King's College Rd., Toronto,

ON M5S 3G8, Canada

e-mail: meguid@mie.utoronto.ca
Keywords COVID-19 - Combating · Superhydrophobic $\cdot$ Self-cleaning $\cdot$ Antibacterial . Antiviral

\section{Introduction}

At the end of 2019, a debilitating and novel strand of coronavirus, known as COVID-19, emerged and progressed into an outbreak (Yang and Wang 2020; Munster et al. 2020). Soon after, the new disease, which put the lives and economic wellbeing of millions at risk, was declared a global pandemic by the World Health Organization (WHO) (Yang and Wang 2020; Munster et al. 2020). Consequently, researchers worldwide devoted significant effort and time to discover a cure or an effective treatment in the form of drugs, vaccines, or antibody therapies. Equally as important is the search for engineering solutions that can help combat or reduce the virus transmission and protect the public and first responders from this infectious disease.

COVID-19 can be transmitted through airborne respiratory droplets, ejected as a result of talking, coughing or sneezing, as well as through human contact with fomites (Yang and Wang 2020; Gralinski and Menachery 2020). Fomites represent a major health concern due to their ability to spread the virus when exposed to contaminated respiratory droplets or 
other body fluids, such as saliva, nasal discharge, or blood, from an infected person (Ong et al. 2020). Recent studies by the National Institute of Allergy and Infectious Diseases (USA) have shown that the virus can infect fomites made of metals, polymers and recycled paper for extended periods measured in days (Van Doremalen et al. 2020). For instance, contaminated commonly used surfaces that exist in public facilities, such as furniture, clothes, utensils, handrails, taps, sinks, elevator buttons, and light switches, among others, can easily transmit the virus. In addition, according to the WHO guidelines (Van Doremalen et al. 2020), Health Care Workers (HCW) should be equipped with personal protective equipment (PPE), such as gowns, coveralls, face shields, or masks, to act as a barrier against fluid transmission and/or fluid penetration. However, infections due to the adhesion of bodily fluids to the surface of PPE during doffing or undressing have previously been reported in (Katoh et al. 2019; Galante et al. 2020; Tanabe et al. 2020), which represents a serious risk of virus transmission among HCW. This risk has been further demonstrated by the numerous COVID-19 infections reported among HCW (Bowdle and Munoz-Price 2020; Wang et al. 2020). It is worth noting that during the SARS outbreak in 2003, 51\% of the cases in Ontario (Canada) were among HCW (Bowdle and MunozPrice 2020). Accordingly, novel strategies should be sought to reduce the risk of virus transmission among the public and first responders.

Superhydrophobic surfaces have recently been garnering considerable attention due to their extreme water repellency features, with contact angles (CA) larger than $150^{\circ}$ and sliding angles (SA) lower than $10^{\circ}$. Additionally, they possess self-cleaning capabilities (Liu et al. 2017; Yan et al. 2011; Teisala and Butt 2018). Due to these highly desirable characteristics, superhydrophobic surfaces have recently gained popularity in the biomedical sector due to their blood repellency and reduction in bacterial, viral adhesion and antifouling properties (Falde et al. 2016; Shin et al. 2016; Jaggessar et al. 2017). Earlier studies have shown that PPE that have high CA and low SA can significantly reduce a virus's carryover potential and provide antimicrobial benefits (Katoh et al. 2019; Tomšič et al. 2008; Yeerken et al. 2019).

For surfaces to acquire superhydrophobicity, a combination of chemical modification and surface texturing is required (Liu et al. 2017; Yan et al. 2011).
Specifically, materials with a low surface energy and contain surfaces with a hierarchical nano/microstructure represent the basis for superhydrophobicity. Although various techniques have been reported in the literature (Yan et al. 2011; Martin et al. 2017), the complexity and investment in the majority of these techniques hinder their widespread adoption into broader commercial applications. A potential fabrication technique that can overcome these obstacles is developing superhydrophobic nanocomposites in which low surface energy nanoparticles are dispersed in a polymeric matrix. These nanocomposites can be applied to a surface to form a superhydrophobic coating (Yeerken et al. 2019; Elzaabalawy et al. 2019; Elzaabalawy and Meguid 2020) or bulk-synthesised to create a monolith that is entirely superhydrophobic (Elzaabalawy et al. 2019; Zhang et al. 2016). The choice of the nanocomposite's constituent materials depends on the required surface characteristics. For instance, flexible polymers with low surface energy, such as PDMS (silicone) and PTFE (Teflon), can be used to coat or create flexible surfaces, while polymers with greater mechanical properties, such as epoxy, can be used to coat commonly accessible and frequently touched surfaces. As for nanoparticles, silica has demonstrated excellent self-cleaning characteristics (Yeerken et al. 2019; Elzaabalawy et al. 2019; Elzaabalawy and Meguid 2020; Zhang et al. 2016) and remains to be a cost effective solution.

The literature indicates that copper can play a crucial role to further eliminate the risk of the virus. Copper fomites were found to have antiviral properties against Influenza A virus particles (Noyce et al. 2007), and are also found to be the fastest to eradicate the activity of COVID-19 virus particles compared to other materials (Van Doremalen et al. 2020), as shown in Fig. 1. Copper-based nanoparticles were also found to inactivate H1N1 influenza virus (Fujimori et al. 2009), as well as other virus' strands (Ravishankar Rai and Jamuna Bai 2011; Shionoiri et al. 2012). Moreover, copper nanoparticles possess antibacterial characteristics (Suryaprabha and Sethuraman 2017; Anita et al. 2011; Agrawal et al. 2019), which can improve the general hygiene level of surfaces. Thus, an effective combating strategy to eliminate the virus would incorporate copper nanoparticles within the nanocomposite or employ separate copper surfaces. 

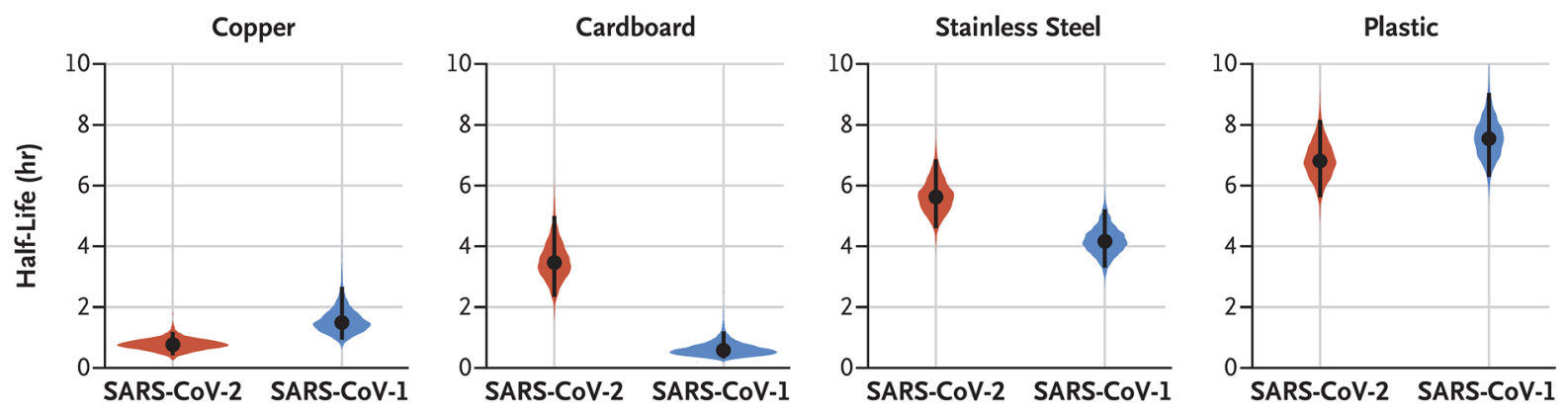

Fig. 1 Half-life of viable virus particles for different materials (Van Doremalen et al. 2020)

\section{Hypothesis}

With the recent COVID-19 virus outbreak, we hypothesise that implementing superhydrophobic surfaces in public and healthcare facilities can protect against and reduce the potential of virus transmission. Since COVID-19 spreads mainly through droplet transmission and via contacting contaminated surfaces, we believe that surfaces coated with or created from a superhydrophobic nanocomposite can combat the virus transmission through a 3-step strategy; as demonstrated in Fig. 2. First, droplets contacting superhydrophobic surfaces will maintain their spherical shape and exist in a Cassie-Baxter wetting state, which reduces the surface's exposure to the virus by encapsulation. Second, we believe that droplets impinging a superhydrophobic surface will either rebound or roll-off leaving no trace of the virus; ultimately resulting in contamination suppression. Finally, for the repelled droplets or in extreme cases where some traces of the virus inevitably contaminate the surface, nanoparticles within the nanocomposite with antiviral and antibacterial properties will eradicate the virus, as shown schematically in Fig. 2.
The superhydrophobic nanocomposites acquire their surface protection characteristics through chemical modification and a hierarchical nano/microstructure. Different polymeric materials can be used to vary the flexibility and endurance/durability of the developed surfaces. Furthermore, a combination of highly hydrophobic particles, such as silica, and other nanoparticles that possess antibacterial and antiviral properties, such as copper, can be used to obtain the desirable surface protection.

\section{Objectives}

Although research and development of novel materials might not provide a direct cure for COVID-19, they can significantly reduce the spread of the virus among the public and first responders. As one of the leading laboratories in superhydrophobicity research, we believe that superhydrophobic surfaces can contribute to the different protection strategies. Specifically, our aim is to design and develop self-cleaning superhydrophobic surfaces either by coating commercially available surfaces or creating monolithic surfaces that

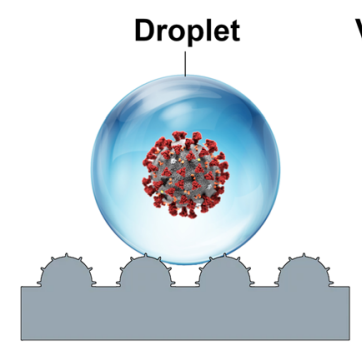

(a)

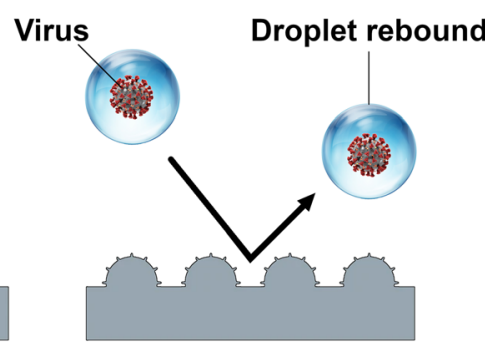

(b)

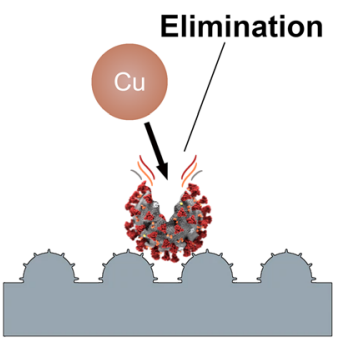

(c)

Fig. 2 A schematic of our proposed transmission combating strategy: a Virus encapsulation, b contamination suppression, and $\mathbf{c}$ virus elimination 
are entirely superhydrophobic. The developed surfaces should be chemically stable, flexible, durable, and safe for all types of applications, including medical applications. In addition, the fabrication method used should be economical, scalable, and facile to ensure their widespread adoption in different applications and specially in healthcare facilities. We have demonstrated these qualities in our earlier work (Elzaabalawy et al. 2019; Elzaabalawy and Meguid 2020) for general engineering applications. The focus here is on the development of durable protective surfaces that are self-cleaning, antibacterial, and antiviral.

\section{Development of superhydrophobic surfaces}

Decidedly, developing superhydrophobic surfaces and monoliths is an effective approach for reducing the transmission potential of COVID-19. Based on the technique reported in our previous work (Elzaabalawy et al. 2019), a flexible superhydrophobic surface can be fabricated by first dispersing hydrophobic nanoparticles, such as silica, in a flexible polymeric matrix, such as silicone. The homogeneous dispersion process can be ensured with the aid of a solvent, such as acetone, and an ultrasonicator. The emulsion produced by the dispersion process can then be applied as a sprayed coating. Consequently, the coated surface is cured at an elevated temperature $\left(120^{\circ} \mathrm{C}\right)$ for $1 \mathrm{~h}$ to ensure its effective polymerization.

In order to fabricate a flexible and regenerative superhydrophobic monolith, the emulsion can alternatively be subjected to a hot-water bath and eccentric mixing to evaporate most of the solvent after homogenous dispersion is achieved. A paste-like mixture is then obtained and can be injected into molds of desired shapes and thicknesses. Upon curing at an elevated temperature $\left(120{ }^{\circ} \mathrm{C}\right.$ for $\left.1 \mathrm{~h}\right)$, a controlled sandpaper abrasion process was used to expose the dispersed nanoparticles and achieve the hierarchical micro/nanostructure necessary for superhydrophobicity. Since the entire bulk of the material is superhydrophobic, a damaged surface can be regenerated by re-applying the abrasion process to restore superhydrophobicity.

The concentration of nanoparticles in the nanocomposite can greatly affect the characteristics of the developed surfaces. It was found that a concentration of $\sim 15 \mathrm{wt} \%$ ensures an optimum balance between superhydrophobicity and endurance for spray coated surfaces. For a superhydrophobic monolith, a concentration of $\sim 10 \mathrm{wt} \%$ yielded excellent repellency features, while at the same time maintaining the viscosity of the emulsion at a value that enables easy injection into molds.

\section{Combating COVID-19 using superhydrophobic surfaces}

Adopting the above concentrations, the developed surfaces can maintain water droplet at a stable CassieBaxter wetting state, with CA of $\sim 163^{\circ}$ and SA of $\sim 5^{\circ}$ for the spray coated surfaces and CA of $\sim 167^{\circ}$ and SA of $\sim 6^{\circ}$ for the regenerative monoliths, as depicted in Fig. 3. Remarkably, the developed surfaces enabled an impinging droplet to roll-off the surface (Fig. 4a, b, d, e), leaving no traces. In fact, even after experiencing a large spread diameter and creating secondary droplets due to the high impinging velocity (Fig. 4c). This exceptional recollection and repellency highlights the capability of the newly developed surface to encapsulate and suppress contaminated surfaces.

SEM investigations have shown that the fabrication technique is capable of creating the hierarchical structure necessary for effecting superhydrophobicity, as shown in Fig. 5. The nanoparticles are responsible for creating the nano-asperities, while the microstructure is obtained as a result of the atomization effect in spray-coated samples and controlled surface abrasion in bulk-synthesized monoliths. The delicate balance between the polymer and the nanoparticles is critical in developing an effective multiscale structure, while maintaining its endurance/durability.

Epoxy has a higher surface energy and as a result superhydrophobic nanocomposites that use epoxy as the base material might experience reduced repellency. Based on our previous work (Elzaabalawy and Meguid 2020), an additional step can be introduced to overcome this reduction. Prior to dispersing the nanoparticles, the epoxy is chemically modified using an amino-functionalized polysiloxane. The silica nanoparticles can then be dispersed in the matrix of siloxane-modified epoxy with the aid of a solvent and ultrasonicator. Subsequently, the spray coated surface would be cured at room temperature for $24 \mathrm{~h}$. It is 


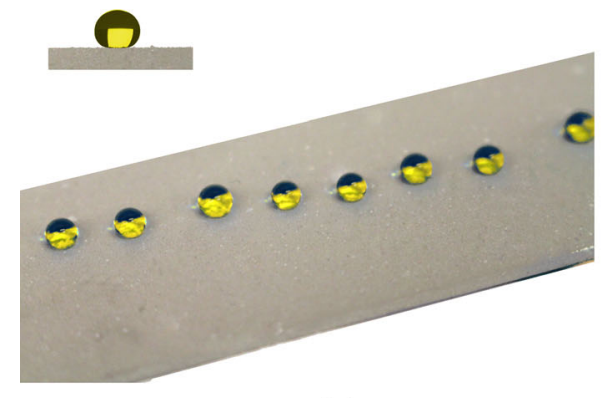

(a)

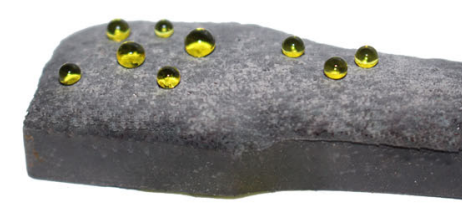

(b)

Fig. 3 Static water droplets (dyed for contrast) on a a spray-coated sample and $\mathbf{b}$ regenerative monolith

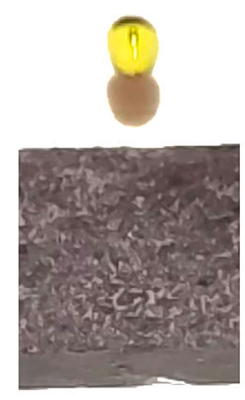

(a)

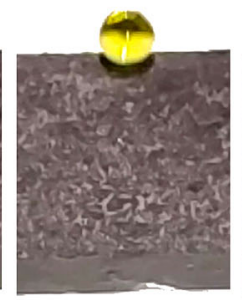

(b)

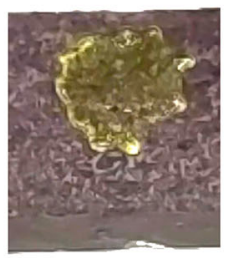

(c)

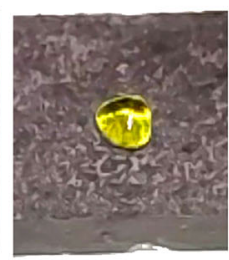

(d)

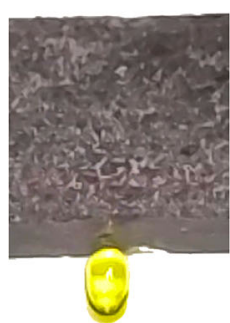

(e)

Fig. 4 Time-lapse images for a droplet impinging a superhydrophobic monolith: a droplet approach, b just prior to impact, $\mathbf{c}$ large spread to droplets, $\mathbf{d}$ droplet reassembly and e roll-off with no traces

worth noting that a concentration of nanoparticles of $\sim 30 \mathrm{wt} \%$ was found to yield a good balance between superhydrophobicity and surface durability.

\subsection{Variety of substrates examined}

The newly developed superhydrophobic coating was also found to be highly effective in treating different substrates: metals, glass, wood, and fabrics, as shown in Fig. 6. Our ability to treat a variety of substrates is crucial in ensuring that the novel coating is capable of providing the necessary protection for different surfaces and applications. Interestingly, coated fabrics demonstrated exceptional repellency features and did not lose their flexibility. This result was attributed to the slight elasticity enhancement achieved through the polysiloxane modification process and the micro- cracks observed within the surface structure of the coating.

Additionally, the coated surfaces enabled impinging droplets $(\sim 10 \mu \mathrm{L})$ to completely rebound within $\sim 16 \mathrm{~ms}$ and completely deflect an impacting water jet, as depicted in Figs. 7 and 8, respectively. It is worth observing that the droplet rebounds leaving no traces on the surface, which is a necessary feature to accomplish the contamination suppression outlined in our strategy to curtail the spread of the virus. Specifically, a surface with a slight inclination will be able to purge any impinging contaminated droplets. The developed hierarchical nano/microstructure of the developed nanocomposite coating is shown clearly in the SEM images depicted in Fig. 9. The presence of nano/micro-asperities superposed on the main surface asperities are one of the main characteristic features of superhydrophobicity. 


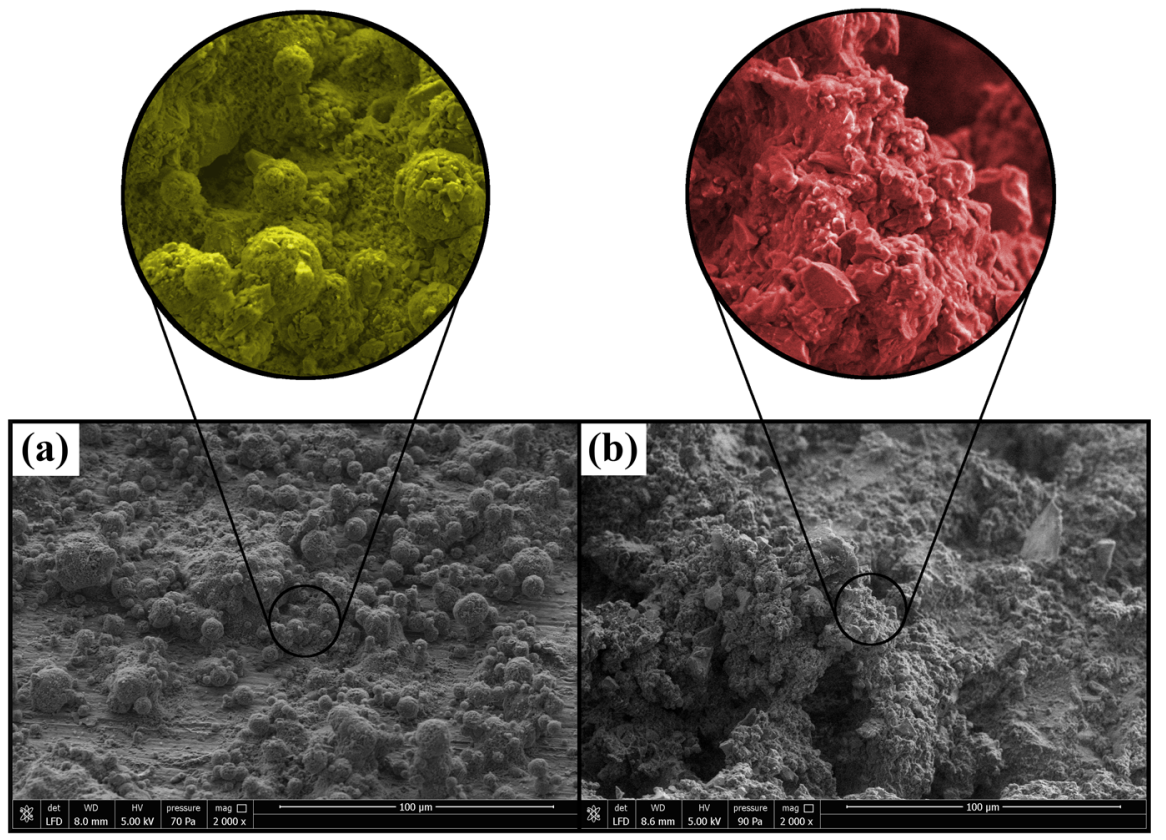

Fig. 5 SEM images for a spray-coated sample and $\mathbf{b}$ regenerative monolith

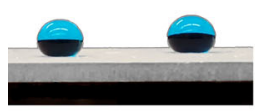

(a)

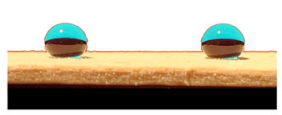

(b)

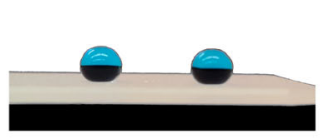

(c)

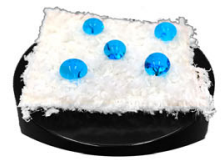

(d)

Fig. 6 Static water droplets on different substrate materials covered with our novel superhydrophobic coating: a metal, b wood, $\mathbf{c}$ glass, and $\mathbf{d}$ fabric

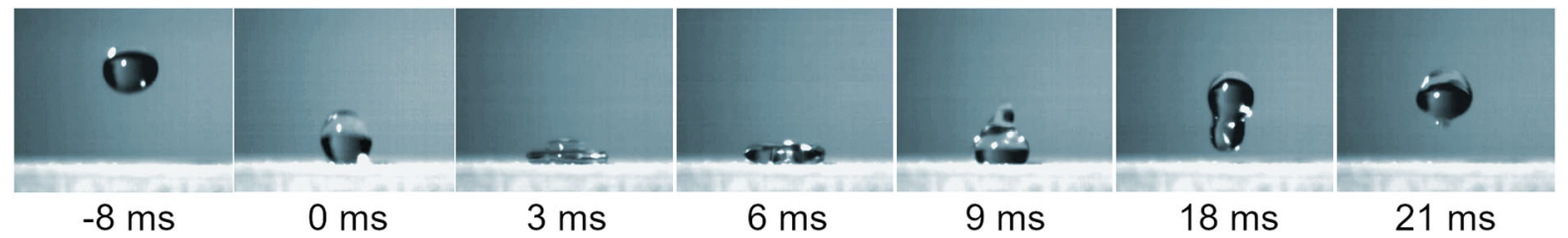

Fig. 7 Typical droplet impingement on a coated metallic sample and rebound at different time intervals leaving no traces

\section{The challenges}

Let us now address some of the challenges and limitations associated with our research. We believe that further enhancement strategies using other nanoparticles that have antibacterial and antiviral characteristics should be pursued. This would ensure the complete eradication of the virus (third feature in our devised combating strategy). Specifically, the surface should first repel contaminated droplets or body fluids to prevent adhesion, keeping the virus encapsulated and supressing the surface contaminants. Further research is also necessary to investigate the potential of copper nanoparticles to target COVID-19 virus strands and the effect of using a blend of highly hydrophobic silica nanoparticles and antibacterial/ 


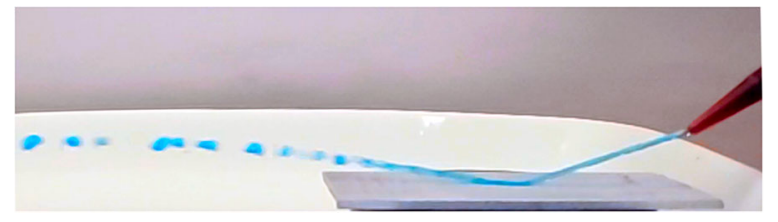

Fig. 8 Repelling an impinging water jet (dyed for contrast) off a metallic coated sample

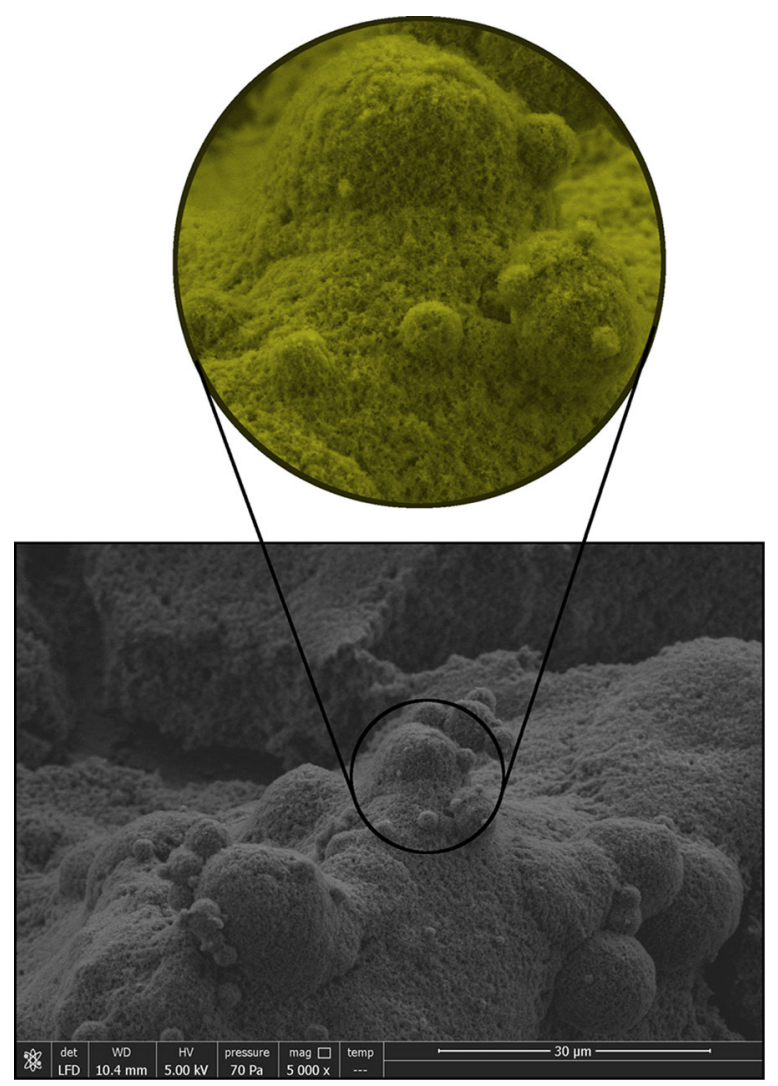

Fig. 9 SEM image for a coated sample

antiviral copper nanoparticles. Additional investigations should also be performed to ensure a balance between superhydrophobicity, durability/endurance, surface protection, and antibacterial/antiviral properties. Clearly, it is necessary to conduct trials using fomites to prove the concepts and the potential of superhydrophobicity in combating the spread of COVID-19. Our laboratory has contacted a number of epidemiology laboratories in the surrounding hospitals to seek their help in testing the ability of our newly developed surfaces to limit the spread of the virus.

\section{Conclusions}

In this work, we highlighted the potential of implementing superhydrophobic surfaces to reduce virus transmission. We succeeded in fabricating nanocomposites that can repel contaminated droplets and provide enhanced protection to surfaces used in public and healthcare facilities. These nanocomposites can be applied to coat surfaces or bulk-synthesized into a superhydrophobic monoliths. Additionally, the mechanical properties of the superhydrophobic nanocomposite can be varied by using different polymeric materials (silicone or epoxy) to suit 
different substrates and operating conditions. We have demonstrated two aspects of our combating strategy: encapsulation and contamination suppression of droplets. Earlier studies have already confirmed the antiviral and antibacterial properties of copper.

Further research is required to investigate the effect of using a mix of highly hydrophobic silica and antibacterial/antiviral copper nanoparticles to obtain an effective surface protection capable of eradicating the virus. Additionally, it is necessary to conduct virus tests using fomites and treated surfaces to demonstrate the concepts discussed herein. With the current uncertain future, the unfortunate loss of life and the devastation of the world economies, we felt obligated to publish our results and make our research known to all interested parties. Clearly, this study does not preclude the importance of social distancing, proper care and hygiene necessary to ensure protection against contracting or spreading the virus.

Acknowledgements This study was financially supported by the Natural Sciences and Engineering Research Council of Canada (Project No. RGPIN-2018-03804).

\section{References}

Agrawal, N., Low, P.S., Tan, J.S.J., Fong, E.W.M., Lai, Y., Chen, Z.: Durable easy-cleaning and antibacterial cotton fabrics using fluorine-free silane coupling agents and $\mathrm{CuO}$ nanoparticles. Nano Mater. Sci. (2019). https://doi.org/10. 1016/j.nanoms.2019.09.004

Anita, S., Ramachandran, T., Rajendran, R., Koushik, C.V., Mahalakshmi, M.: A study of the antimicrobial property of encapsulated copper oxide nanoparticles on cotton fabric. Text. Res. J. 81, 1081-1088 (2011). https://doi.org/10. 1177/0040517510397577

Bowdle, A., Munoz-Price, L.S.: Preventing infection of patients and healthcare workers should be the new normal in the era of novel coronavirus epidemics. Anesthesiology (2020). https://doi.org/10.1097/aln.0000000000003295

Elzaabalawy, A., Meguid, S.A.: Development of novel superhydrophobic coatings using siloxane-modified epoxy nanocomposites. Chem. Eng. J. (2020). https://doi.org/10. 1016/j.cej.2020.125403

Elzaabalawy, A., Verberne, P., Meguid, S.A.: Multifunctional silica-silicone nanocomposite with regenerative superhydrophobic capabilities. ACS Appl. Mater. Interfaces 11, 42827-42837 (2019). https://doi.org/10.1021/acsami. 9b15445

Falde, E.J., Yohe, S.T., Colson, Y.L., Grinstaff, M.W.: Superhydrophobic materials for biomedical applications. Biomaterials 104, 87-103 (2016). https://doi.org/10.1016/j. biomaterials. 2016.06 .050
Fujimori, Y., Sato, T., Hayata, T., Nagao, T., Nakayam, M., Nakayam, T., Sugamat, R., Suzuki, K.: Novel antiviral characteristics of nanosized copper(i) iodide particles showing inactivation activity against 2009 pandemic H1N1 influenza virus. Appl. Environ. Microbiol. 78(2012), 951-955 (2009). https://doi.org/10.1128/AEM.06284-11

Galante, A., Haghanifar, S., Romanowski, E.G., Shanks, R., Leu, P.W.: Superhemophobic and anti-virofouling coating for mechanically durable and wash-stable medical textiles. ACS Appl. Mater. Interfaces (2020). https://doi.org/10. 1021/acsami.9b23058

Gralinski, L.E., Menachery, V.D.: Return of the coronavirus: 2019-nCoV. Viruses 12, 1-8 (2020). https://doi.org/10. 3390/v12020135

Jaggessar, A., Shahali, H., Mathew, A., Yarlagadda, P.K.D.V.: Bio-mimicking nano and micro-structured surface fabrication for antibacterial properties in medical implants. J. Nanobiotechnol. 15, 1-20 (2017). https://doi.org/10. 1186/s12951-017-0306-1

Katoh, I., Tanabe, F., Kasai, H., Moriishi, K., Shimasaki, N., Shinohara, K., Uchida, Y., Koshiba, T., Arakawa, S., Morimoto, M.: Potential risk of virus carryover by fabrics of personal protective gowns. Front. Public Heal. 7, 3-8 (2019). https://doi.org/10.3389/fpubh.2019.00121

Liu, M., Wang, S., Jiang, L.: Nature-inspired superwettability systems. Nat. Rev. Mater. (2017). https://doi.org/10.1038/ natrevmats.2017.36

Martin, S., Brown, P.S., Bhushan, B.: Fabrication techniques for bioinspired, mechanically-durable, superliquiphobic surfaces for water, oil, and surfactant repellency. Adv. Colloid Interface Sci. 241, 1-23 (2017). https://doi.org/10.1016/j. cis.2017.01.004

Munster, V.J., Koopmans, M., van Doremalen, N., van Riel, D., de Wit, E.: A novel coronavirus emerging in China-key questions for impact assessment. N. Engl. J. Med. 382, 692-694 (2020). https://doi.org/10.1056/NEJMp2000929

Noyce, J.O., Michels, H., Keevil, C.W.: Inactivation of influenza A virus on copper versus stainless steel surfaces. Appl. Environ. Microbiol. 73, 2748-2750 (2007). https:// doi.org/10.1128/AEM.01139-06

Ong, S.W.X., Tan, Y.K., Chia, P.Y., Lee, T.H., Ng, O.T., Wong, M.S.Y., Marimuthu, K.: Air, surface environmental, and personal protective equipment contamination by Severe Acute Respiratory Syndrome Coronavirus 2 (SARS-CoV2) from a symptomatic patient. JAMA J. Am. Med. Assoc. 323, 1610-1612 (2020). https://doi.org/10.1001/jama. 2020.3227

Ravishankar Rai, V., Jamuna Bai, A.: Nanoparticles and their potential application as antimicrobials. In: Méndez-Vilas, A. (ed.) Science Against Microbial Pathogens: Communicating Current Research and Technological Advances. Formatex, Microbiology Series, vol. 1, pp. 197-209. Spain (2011)

Shin, S., Seo, J., Han, H., Kang, S., Kim, H., Lee, T.: Bioinspired extreme wetting surfaces for biomedical applications. Materials (Basel) (2016). https://doi.org/10.3390/ ma9020116

Shionoiri, N., Sato, T., Fujimori, Y., Nakayama, T., Nemoto, M., Matsunaga, T., Tanaka, T.: Investigation of the antiviral properties of copper iodide nanoparticles against 
feline calicivirus. J. Biosci. Bioeng. 113, 580-586 (2012). https://doi.org/10.1016/j.jbiosc.2011.12.006

Suryaprabha, T., Sethuraman, M.G.: Fabrication of copperbased superhydrophobic self-cleaning antibacterial coating over cotton fabric. Cellulose 24, 395-407 (2017). https:// doi.org/10.1007/s10570-016-1110-Z

Tanabe, F., Uchida, Y., Arakawa, S., Morimoto, M.: Increased adhesion of methicillin-resistant Staphylococcus aureus to the surface of personal protective clothing damaged by friction during nursing action. Am. J. Infect. Control 48, 416-419 (2020). https://doi.org/10.1016/j.ajic.2019.08. 028

Teisala, H., Butt, H.J.: Hierarchical structures for superhydrophobic and superoleophobic surfaces. Langmuir (2018). https://doi.org/10.1021/acs.langmuir.8b03088

Tomšič, B., Simončič, B., Orel, B., Černe, L., Tavčer, P.F., Zorko, M., Jerman, I., Vilčnik, A., Kovač, J.: Sol-gel coating of cellulose fibres with antimicrobial and repellent properties. J. Sol-Gel. Sci. Technol. 47, 44-57 (2008). https://doi.org/10.1007/s10971-008-1732-1

Van Doremalen, N., Bushmaker, T., Morris, D., Holbrook, M., Gamble, A., Williamson, B., Tamin, A., Harcourt, J., Thornburg, N., Gerber, S., Lloyd-Smith, J.: Aerosol and surface stability of SARS-CoV-2 as compared with SARSCoV-1. N. Engl. J. Med. 382(16), 1564-1567 (2020)

Wang, J., Zhou, M., Liu, F.: Reasons for healthcare workers becoming infected with novel coronavirus disease 2019
(COVID-19) in China. J. Hosp. Infect. (2020). https://doi. org/10.1016/j.jhin.2020.03.002

Yan, Y.Y., Gao, N., Barthlott, W.: Mimicking natural superhydrophobic surfaces and grasping the wetting process: a review on recent progress in preparing superhydrophobic surfaces. Adv. Colloid Interface Sci. 169, 80-105 (2011). https://doi.org/10.1016/j.cis.2011.08.005

Yang, P., Wang, X.: COVID-19: a new challenge for human beings. Cell. Mol. Immunol. (2020). https://doi.org/10. 1038/s41423-020-0407-x

Yeerken, T., Wang, G., Li, H., Liu, H., Yu, W.: Chemical stable, superhydrophobic and self-cleaning fabrics prepared by two-step coating of a polytetrafluoroethylene membrane and silica nanoparticles. Text. Res. J. 89, 4827-4841 (2019). https://doi.org/10.1177/0040517519842795

Zhang, X., Zhu, W., He, G., Zhang, P., Zhang, Z., Parkin, I.P.: Flexible and mechanically robust superhydrophobic silicone surfaces with stable Cassie-Baxter state. J. Mater. Chem. A 4, 14180-14186 (2016). https://doi.org/10.1039/ c6ta06493k

Publisher's Note Springer Nature remains neutral with regard to jurisdictional claims in published maps and institutional affiliations. 